\title{
Electron Microscopic Characterization of a Gelatin Matrix/Thrombin Hemostat
}

\author{
J.P. DiOrio*, L. Stojanovic*, A. Yardimci*, D.L. Amrani*, S. Helgerson**, F. Vega** \\ * Baxter Technology Resources, Route 120 and Wilson Road, Round Lake, Illinois 60002 \\ ** Baxter Bioscience, 34175 Ardenwood Blvd, Fremont, California 94555
}

FloSeal ${ }^{\circledR}$ Matrix Hemostatic Sealant (Baxter Healthcare Corp.) is composed of a cross-linked gelatin matrix combined with thrombin to produce a highly viscous gel that functions as a topical hemostatic agent [1]. FloSeal ${ }^{\circledR}$ is approved for use in surgery as an adjunct treatment when control of bleeding by conventional procedures is ineffective or impractical [2]. Both the gelatin matrix and the thrombin (King Pharmaceuticals, Inc.) are of bovine origin in the current Floseal ${ }^{\circledR}$ product. The present study characterizes the structure of the cross-linked gelatin particles and the associated clot formed in vivo using bovine thrombin, and then, compares the in vivo clot structures seen when the bovine thrombin component is replaced by a human-derived thrombin (Baxter Healthcare Corp.).

A pig (Yorkshire/Hampshire strain) was anesthetized and prepared for surgery under a protocol approved by the Institutional Animal Care and Use Committee of the test facility. A midline laparotomy was performed using standard surgical techniques, and the liver exposed and isolated. A blood sample was taken and Activated Clotting Time (ACT) recorded. The animal's ACT was then maintained at a level of 3 to 5 times this baseline by an initial intravenous dose of heparin sodium at $250 \mathrm{IU} / \mathrm{kg}$ weight, followed by doses of $125 \mathrm{IU} / \mathrm{kg}$ as needed, throughout the experiment. A series of square lesions, approximately $1 \mathrm{~cm} \times 1 \mathrm{~cm} \times 0.3 \mathrm{~cm}$, was created on the liver surface using sharp dissection. The lesions were then treated by filling with FloSeal test materials. The test materials were held in gentle approximation to the lesion for two minutes using a saline-moistened gauze sponge. The gauze was then removed. This treatment was sufficient to achieve hemostasis in all lesions. The animal was euthanized per facility guidelines at the end of the experiment. The lesions were removed en-bloc, taking care to not disturb the test materials and fixed in $2 \%$ glutaraldehyde.

Samples for scanning electron microscopy (SEM) were dehydrated through a graded $0-100 \%$ ethanol series and air-dried out of Hexamethyldisilazane. They were coated with palladium and imaged on a JEOL 6300 field emission SEM operated at 5kV. Samples for Transmission electron microscopy (TEM) were osmicated followed by dehydration through a graded $0-100 \%$ ethanol series into acetone and embedment in Spurr's resin. Thin sections were stained with uranyl acetate and lead citrate and were imaged on a JEOL 1200 EX TEM operated at 60kV.

The in vivo clot structures formed using the combination of cross-linked gelatin particles and bovine thrombin (IIa) are shown in Figures 1 - 4. The cross-linked gelatin particles with associated clot are shown in Fig. 1. An area between the gelatin particles filled with a dense fibrous material entrapping blood cells is shown at higher magnification in Fig. 2. Red blood cells (RBC) on the surface of an individual gelatin particle are shown in Fig. 3. The inset shows the fibrous material covering the surface of another gelatin particle. The ultrastructure of a cross section from a similar area also shows this fibrous material between the RBC and a gelatin particle. The individual fibers in an adjacent area had a $225 \AA$ repeat pattern characteristic of fibrin fibers (not shown). Figures 5 6 show the structure of an in vivo clot formed using the cross-linked gelatin particles and human thrombin. Similar structures were seen in SEM for both the bovine and human thrombin samples. 
References

[1] C. Mehmet, et al., J. Card. Surg. 18 (2003) 486.

[2] C. Mehmet et al., Ann. Thorac. Surg. 69 (2000) 1376.
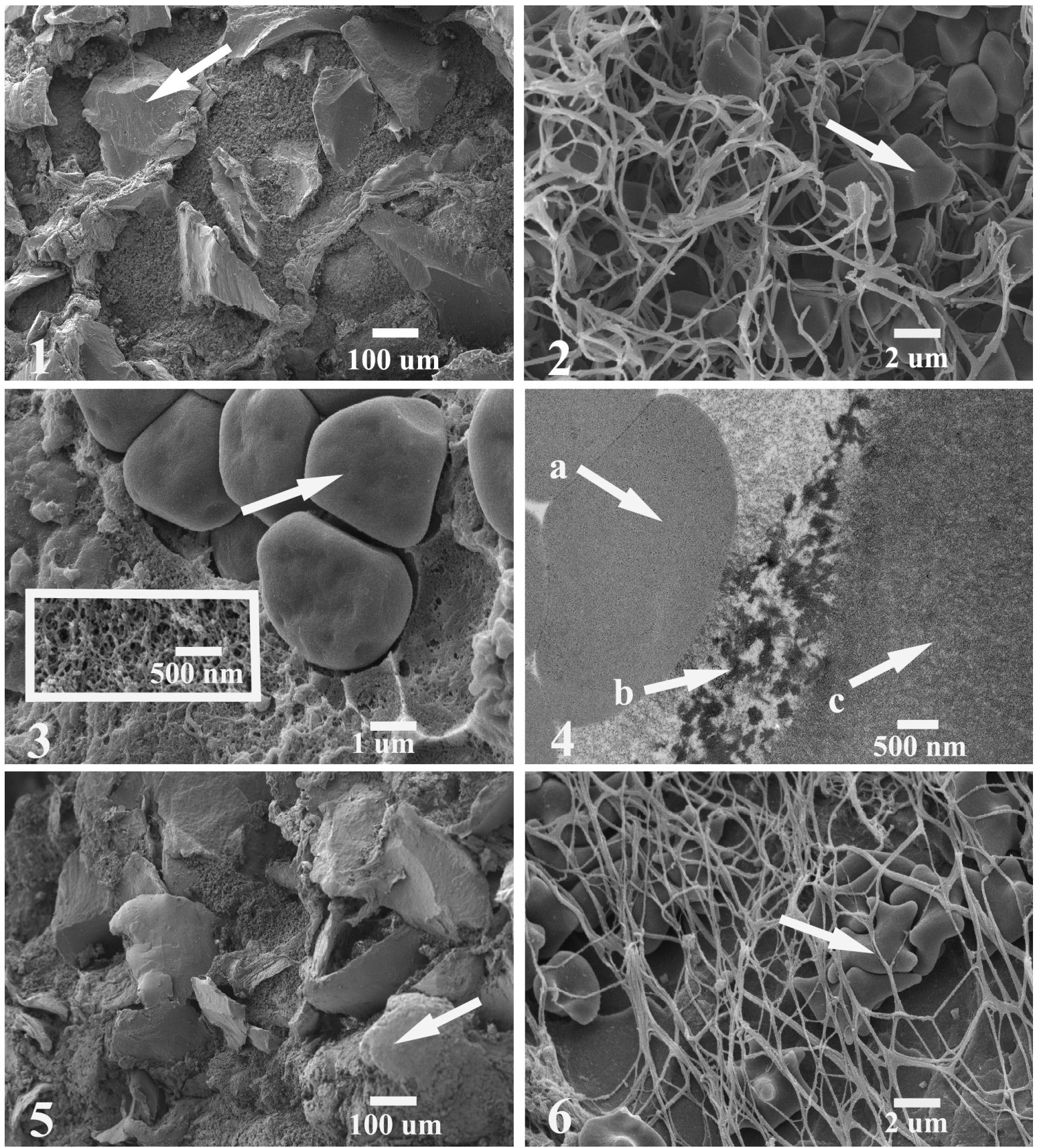

Fig. 1. SEM of gelatin particles/IIa (arrow) with associated blood cells and clot (bovine thrombin). Fig. 2. SEM of an area between the gelatin particles with a fibrin clot trapping blood cells (arrow). Fig. 3. SEM of a particle surface with RBC's (arrow). Inset: surface of another gelatin particle. Fig. 4. TEM of similar area in Fig. 3. Arrows: a) RBC b) fibrous material c) gelatin bead. Fig. 5. SEM of gelatin particles/IIa (arrow) with associated blood cells and clot (human thrombin). Fig. 6. SEM of an area between the gelatin particles with a fibrin clot trapping blood cells (arrow). 